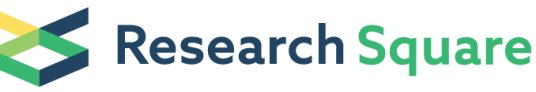

\section{Prevalence of anti-SARS-CoV-2 antibodies among blood donors in South Africa during the period January-May 2021}

Marion Vermeulen ( $\sim$ Marion.Vermeulen@sanbs.org.za)

South African National Blood Service https://orcid.org/0000-0003-4383-4526

Laurette Mhlanga

Stellenbosch University https://orcid.org/0000-0002-7805-4231

\section{Wendy Sykes}

South African National Blood Service

\section{Charl Coleman}

South African National Blood Service https://orcid.org/0000-0003-3272-927X

\section{Nadia Pietersen}

Western Cape Blood Service

\section{Russell Cable}

Western Cape Blood Service

\section{Ronel Swanevelder}

South African National Blood Service

\section{Tanya Nadia Glatt}

South African National Blood Service https://orcid.org/0000-0002-5458-5791

\section{Eduard Grebe}

Vitalant Research Institute https://orcid.org/0000-0001-7046-7245

\section{Alex Welte}

South African DST-NRF Centre of Excellence in Epidemiological Modelling and Analysis, Stellenbosch University https://orcid.org/0000-0001-7139-7509

\section{Karin van den Berg}

South African National Blood Service https://orcid.org/0000-0001-9805-8013

\section{Research Article}

Keywords: COVID-19, seroprevalence, anti-SARS-CoV-2 antibodies, blood donors, South Africa

Posted Date: August 2nd, 2021

DOI: https://doi.org/10.21203/rs.3.rs-690372/v2 
License: (c) (i) This work is licensed under a Creative Commons Attribution 4.0 International License. Read Full License 


\title{
Prevalence of anti-SARS-CoV-2 antibodies among blood donors in South Africa during the period January-May 2021
}

\author{
6 July 2021
}

\begin{abstract}
Marion Vermeulen ${ }^{1,7}$, Laurette Mhlanga ${ }^{2}$, Wendy Sykes $^{1}$, Charl Coleman ${ }^{1}$, Nadia Pieterson ${ }^{3}$, Russell Cable $^{3}$, Ronel Swanevelder ${ }^{1}$, Tanya Nadia Glatt ${ }^{1}$, Eduard Grebe ${ }^{2,4,5}$, Alex Welte ${ }^{2}$, Karin van den Berg $1,6,7$
\end{abstract}

Affiliations: 1) South African National Blood Service, 2) DSI-NRF Centre of Excellence in Epidemiological Modelling and Analysis (SACEMA), Stellenbosch University 3) Western Cape Blood Service, 4) Vitalant Research Institute, 5) University of California San Francisco, 6) University of Cape Town, 7) University of the Free State

\section{Abstract}

Background: Population-level estimates of the prevalence of anti-SARS-CoV-2 antibody positivity (seroprevalence) are crucial epidemiological indicators for tracking the Covid-19 epidemic. Such data are in short supply, both internationally and in South Africa. The South African blood services (the South African National Blood Service, SANBS and the Western Cape Blood Service, WCBS) are coordinating nationwide surveillance of blood donors.

Methods: Leveraging existing arrangements, SANBS human research ethics committee permission was obtained to test blood donations collected on predefined days (in January and May 2021) for anti-SARS-CoV-2 antibodies, using the Roche Elecsys Anti-SARS-CoV-2 assay on the cobas e411 and e801 platforms currently available in the blood services' donation testing laboratories. Using standard methods, prevalence analysis was done by province, age, time, sex and race.

Results: We report on data from 16762 donations. Prevalence varied substantially across race groups and between provinces, with seroprevalence among Black donors consistently several times higher than among White donors, with the other main population groups (Coloured and Asian) not well represented in all provinces. There is no clear evidence that seroprevalence among donors varies by age or sex. The weighted national estimate of prevalence (in the core age range 15-69 years) is $47.4 \%$ ( $95 \% \mathrm{Cl} 46.2-48.6)$. From January to May, we noted a slight but statistically insignificant increase in seroprevalence in those provinces (Gauteng and Free State) where sufficient data were available to make such an estimate.

Conclusions: Our study demonstrates substantial differences in dissemination of SARS-CoV-2 infection between different race groups and provinces, in patterns consistent with known differences in historically entrenched socio-economic status and housing conditions. As has been seen in other contexts, even such high seroprevalence does not guarantee population-level immunity against new outbreaks, as evidenced by a substantial third wave that has emerged almost contemporaneously with the end of sampling in this study. The relative importance of various contributions to this resurgence (notably viral evolution, waning of antibody neutralization efficacy, and infection control fatigue) are unclear. Despite its limitations, notably a 'healthy donor' effect and the possible waning of detectable antibodies over the time scale of the COVID-19 pandemic, it seems plausible that these estimates are reasonably generalisable to actual population level antiSARS-CoV-2 seroprevalence. The interpretation of occasional seroprevalence surveys as a proxy for total attack rates, over the ever-lengthening pandemic time scale is likely to become ever more complex. More frequent sampling, including linked repeat observations of frequent donors, could substantially improve the utility of blood donor surveillance. 


\section{Introduction}

Coronavirus disease 2019 (Covid-19) caused by the virus SARS-CoV-2, manifests in a plethora and range of symptoms, varying from asymptomatic to severe disease which may lead to death. It is this range of severity as well as limited access to health care that makes it difficult to determine how many people have been infected with the virus. After contracting SARS-CoV-2, the majority of people will develop antibodies as part of their immune response. These antibodies last from between 6 and 12 months and can therefore provide an indication of the number of people who have been infected during that time. Given the substantial uncertainties around the true counts of cases of SARS-CoV- 2 infection, and prior studies indicating that in many settings the confirmed case count is only a small proportion of all laboratory confirmed infections, it is of ongoing importance to obtain credible estimates of the prevalence of anti-SARS-CoV-2 antibody positivity (seroprevalence), at the community level $(1,2)$.

\section{Methods}

The South African National Blood Service (SANBS, serving 8 of 9 provinces in South Africa) and Western Cape Blood Service (WCBS, servicing the Western Cape) obtained ethics clearance from the SANBS Human Research Ethics Committee to perform a SARS-CoV-2 seroprevalence study among South African blood donors. The protocol allowed for the testing of routinely collected donor screening samples on predefined 'collection days' in January, March and May; which were internally communicated to blood centre staff at participating collection sites, but without prior notice to potential donors. All donors underwent routine screening through a self-administered questionnaire, one-on-one assessment and a mini-health screening by blood centre staff. Donors who did not meet the routine donor eligibility criteria were excluded from donation and therefore from the study. Contact with persons infected by COVID-19, unresolved COVID-19 infection or COVID-19-like symptoms in the preceding 14 days resulted in temporary deferral of potential donors

Samples collected at the time of donation were tested for anti-SARS-CoV-2 antibodies, using the Roche Elecsys Anti-SARS-CoV-2 total immunoglobulin nucleocapsid assay on the cobas e411 and e801 platforms already in use at the blood services. This assay, according to the package insert, has diagnostic specificity in excess of $99.5 \%$, and near perfect sensitivity (point estimate of $100 \%$ ) at 16 days post PCR positivity. It detects only anti-nucleocapsid antibodies, and so does not detect antibodies mounted in response to any of the vaccines in use, which only present (and stimulate production of antibodies against) viral spike proteins. We do not here explore various nuances of how to define and estimate test performance characteristics by distribution of cases (defined primarily by severity of infection and time since infection/symptoms/PCR detection), but we note:

- Sensitivity and specificity 'in our hands' was investigated by testing 618 samples from the pre-COVID-19 era (1 marginal false positive precisely at the diagnostic threshold) and 50 samples confirmed as positive in a COVID-19 convalescent plasma study protocol (with 1 false negative).

- For epidemiological interpretation, we take seroprevalence as a close proxy of the prevalence of having been infected with SARS-CoV-2 at some point. The Elecsys Anti-SARSCoV-2 assay appears to have particularly good durability of antibody detection for months post PCR reversion and symptom resolution, with no evidence of antibody waning and seroreversion over more than four months in a US COVID-19 convalescent plasma cohort (9). 
- We ignore, for now, the effects of 1) the donor deferral rule that people with confirmed SARS-CoV-2 infection, or COVID-19-like symptoms, are precluded from donation for a period of two weeks after PCR test and/or symptom resolution, and 2) deferral of regular donors who were in quarantine due to a positive contact, and who therefore skipped their routine donation. Given the high rate of asymptomatic infection, this is a relatively minor limitation.

We did not perform structured sampling in the sense of selecting a subset of donation sites or regions within a province. The study merely observed all consenting donors who happened to present themselves at any donation facility on collection days.

Prevalence was estimated by typical categorical and continuous predictors (age, sex, race and province) by standard methods, using the R platform for statistical computation. Although we are not aware of any biological basis for expecting racial differences - in South Africa, as elsewhere, race is, for historical reasons, a strong correlate of socio-economic status, living conditions, and social circumstances, and therefore a suspected predictor of prevalence. As freely downloadable data sets from Statistics South Africa do not disaggregate sufficiently for our purposes, our provincial weighted seroprevalence estimates are based on population size estimates from Machemedze et al (3), interpolated to March 2021, and a racial breakdown of provinces as observed in the 2011 census (4). The level of (dis)aggregation for headline estimates was chosen based on the results of exploratory analysis, as reported below.

Each province was sampled primarily in either January or May, with only Gauteng (GP) and Free State (FS) having a statistically meaningful number of specimens from another month (GP - January, FS - May). To understand the time dimension in our data, we performed a regression in which the data for White and Black donors, from the FS and from GP, was fitted to a model that assigns each of the four subgroups their own prevalence, but with an exponential time dependence that is governed by a single universal rate shared by both provinces and race groups.

\section{Results}

The demographic breakdown of the sampled donors is displayed in figure 1 and tabulated in Table 1. There were slightly more male donors (51.2\%). The large majority of donors in our study were White (51.4\%) and Black (35.6\%) with the remainder distributed mainly between donors self-identifying as Asian (4.2\%) and 'Coloured' (8.1\%) - a uniquely South African racial label indicating persons with a significant mix of ancestry from, amongst other lineages, South Asia, Indonesia, Southern Africa and Europe (5). Only $0.8 \%$ did not report a racial identification. Figure 1 shows the age distribution of donors included in the present analysis, further decomposed by race and province. The provincial totals are shown in Table 2.

After categorizing by either broad or narrow age bins in all provinces and the major race groups, there was no association between seroprevalence and age (see Figure $2 \mathrm{a}$ and $2 \mathrm{~b}$ for broad age bins). There was no association between seroprevalence and sex. See Figure 3 for disaggregation by sex, race and province. Therefore, for the remaining analysis, we do not disaggregate by either age or sex. The regression of data from GP and FS against time provided an estimate of a (relative) $1.6 \%$ per month growth in prevalence, which, at a $p$ value of 0.3 , is not statistically significant, but is large enough (and in the right direction) to be consistent with the crude growth in case detections, in the absence of seroreversion. 
Figure 4 shows the seroprevalence estimates by the remaining meaningful disaggregation - race and province. The large difference by both race and province are highly statistically significant as well as epidemiologically meaningful. Note also the race-weighted overall provincial prevalence estimates (which we interpret as provincial 'attack rates'), and the official prevalence of having been diagnosed, based on reporting of positive PCR diagnostic test results, according to the National Institute for Communicable Diseases (NICD) (6) in the dominant month of sampling: January for the Eastern Cape (EC), Free State FS, Northern Cape (NC), KwaZulu Natal (ZN); May for Gauteng (GP), Limpopo (LP), Mpumalanga (MP), Northwest (NW), Western Cape (WC). The NICD reports on testing performed both in the private and public sector.

Table 3 shows our provincial estimates of attack rates, as a percentage; the implied number of infections; the number of laboratory confirmed cases according to the NICD (6); and the (multiplicative) discrepancy between our estimate and the official count. Note that our estimated number of infections is conservatively based on our estimated prevalence being applied only to the age group 15-69, so these factors are not quite as large as implied by Figure 4 . The estimated seroprevalence ranges from $31.8 \%$ in NC to $62.5 \%$ in the EC and ranges from 6 (WC) to 26 (LP) fold higher than the official case count.

\section{Discussion}

Our study confirms high seroprevalence rates, particularly among Black donors, with little sign of significant population level immunity among other race groups. These substantial differences most likely can be explained by historically based socio-economic factors which hinder the implementation of COVID-19 preventative measures at a community level. The generally high levels of seroprevalence across the whole country are consistent with expectations, given the high burdens experienced on the health care system, and generally low proportion of SARS-CoV- 2 infections which present as serious illness.

Previous seroprevalence estimates from South Africa, specifically the WC already found, before the second wave: 1 ) a very high prevalence (30-40 percent) among pregnant women attending state sector antenatal care, and people living with HIV presenting for routine viral load assessment (7); and 2) higher prevalence among workers with lower socioeconomic status (8). A household cohort study performed in a rural setting in Mpumalanga and an urban setting in North West province found a seroprevalence of $7 \%(95 \% \mathrm{Crl} 5-9 \%)$ and $27 \%(95 \% \mathrm{Crl} 23-31 \%)$, after the first wave of infection, and $26 \%(95 \% \mathrm{Crl} 22-29 \%)$ and $41 \%(95 \% \mathrm{Crl} 37-45 \%)$ respectively after the second wave (10).

For an indication of the meaning of such high seroprevalence values, in a one year old epidemic, consider: a prevalence of $50 \%$, accumulated over 50 weeks, of a condition with a duration of infectiousness of 1 week, implies an average 'prevalence of infectiousness' of $1 \%$ of the population, with inevitable significant elevations above this average value during peaks. For people reliant on public transport, or working in public spaces, it will be difficult to limit close encounters to fewer than 100 people on any given day - i.e. it will be difficult to encounter fewer than one infectious person per day.

We do not claim that blood donors are perfectly representative of the South African population. Firstly, Black and White donors each account for roughly half the total participants of this study, though South Africa's population is about 80 percent Black African and only 8 percent White/European (4). Other population groups are generally insignificantly small except Asian in ZN 
(about 20\%) and Coloured in the WC (about 50\%). Of course, our analysis explicitly weights for racial representativeness. The age weighting we adopted to estimate total infections also produces a face value underestimate for population totals, as it assigns no cases in the age range 0-14 years, which accounts for about 30 percent of the population. Furthermore, repeat blood donors (who supply the majority of donations) are pre-selected to have recently been negative for pathogens included in routine blood safety screening. In South Africa this selection for being HIV negative is certainly relevant, given the country's extraordinary HIV prevalence. Communities which are economically stressed, or without ease of access to blood donor centres, will be under-represented among the study population.

Survey dates represented in this analysis are either:

- barely past South Africa's 'second wave' in COVID-19 incidence - whence deferral rules based on confirmed infection or COVID-19-like symptoms should slightly depress seroprevalence estimates relative to 'true' prevalence; or

- shortly before the emergence of the 'third wave' - whence the interpretation of all these samples, as being from a fairly well-defined epidemiological stage, is not entirely unreasonable.

The Elecsys Anti-SARS-CoV-2 antibody assay appears to have particularly good detection sensitivity for months post PCR reversion (9), though there may be some seroreversion. Therefore, while further investigation of the issue of representativeness will clearly need to be done, our estimates are subject to downward bias by at least some obvious considerations.

With due consideration to both the patent and latent limitations of our study, the key observations we wish to make at this point are:

- The particularly high attack rates in majority Black communities points to the limitations, thus far, of non-pharmaceutical interventions in the context of economic deprivation and high population density, and the urgency of making vaccines available in all communities.

- The high seroprevalence (especially amongst Black donors) also raises interesting and important questions about the level of collective immunity thus far obtained through the two primary infection waves to date - but we caution against simplistic interpretations, given that substantial outbreaks have been seen in cities after the observation of very high seroprevalence (11), and more recent concerns about vaccine efficacy against new variants.

- The low seroprevalence amongst White donors suggests that predominantly White suburban communities lack meaningful collective immunity, and should take infection control measures very seriously for the foreseeable future, especially at the time of writing, when the third wave is presenting many communities with rapidly increasing incidence.

- Given the relatively low marginal cost of leveraging the infrastructure of the blood services, we are keen to further probe the representativeness of blood-donor-based seroprevalence surveys, and to see to what extent surveillance in the blood services can be a valuable and efficient ongoing activity during major infectious disease outbreaks. 


\section{Acknowledgements}

The authors would like to thank the many blood donors who give of their time and lifeblood for the benefit of others, without material reward, while also consenting to the research applications of their specimens. We thank the many nurses and logistical and laboratory staff without whom there would be no transfusion service, no safety screening, and no research project. Additionally, we thank numerous colleagues who have helped us clarify our thinking about the issues involved, including ethical, operational and analytical aspects.

Funding for the operational activities was provided by the South African National Blood Service and the Western Cape Blood Service in part supported by the South African Medical Research Council with funds received from the Department of Science and Innovation, as well as the ELMA South Africa Foundation (Grant number 20-ESA011). The time of investigators in the blood services is supported by the blood services. Karin van den Berg and Marion Vermeulen are also supported by the NIH Fogarty International Center training grant 1D43-TW010345. Alex Welte and Laurette Mhlanga are supported by a Centre of Excellence grant from the South African Department of Science and Innovation via the National Research Foundation. Eduard Grebe is supported by internal funding from Vitalant Research Institute, San Francisco.

\section{Conflicts of Interest}

The Authors affirm they have no conflicts of interest with regard to this publication. 


\section{References}

1. Goudsmit J. The paramount importance of serological surveys of SARS-CoV-2 infection and immunity. Eur J Epidemiol. 2020;35(4):331-3. Available from:

https://doi.org/10.1007/s10654-020-00635-2

2. Lai CC, Wang JH, Hsueh PR. Population-based seroprevalence surveys of anti-SARS-CoV-2 antibody: An up-to-date review. Int J Infect Dis. 2020;101:314-22. Available from: https://doi.org/10.1016/j.ijid.2020.10.011

3. Machemedze T, Kerr A, Dorrington R. WIDER Working Paper 2020/67-South African population projection and household survey sample weight recalibration. 2020 [cited 2021 Feb 7]; Available from: https://doi.org/10.35188/UNU-WIDER/2020/824-5

4. Total population of South Africa 2018, by ethnic groups: Statista Research Department; Nov 3, 2020. Available from: https://www.statista.com/statistics/1116076/total-population-ofsouth-africa-by-population-group/

5. Daya M, Der Merwe L, Galal U, Möller M, Salie M, Chimusa ER, et al. A panel of ancestry informative markers for the complex five-way admixed South African Coloured population. PLoS One. 2013;8(12).

6. NICD. COVID-19 weekly epidemiology brief. 2021. p. 1. Available from: https://www.nicd.ac.za/wp-content/uploads/2021/01/COVID-19-Weekly-Epidemiology-Briefweek-2-2021.pdf

7. Hsiao $\mathrm{M}$ et al, SARS-CoV-2 seroprevalence in the cape town metropolitan sub-districts after the peak of infections. COVID-19 Special Public Health Surveillance Bulletin, 18 (S5)

8. Shaw et al Higher SARS-CoV-2 Seroprevalence in Workers with Lower Socioeconomic Status in Cape Town, South Africa. Research Square, DOI: 10.21203/rs.3.rs-136543/v1

9. Busch MP. private communication (Feb 2021).

10. Kleynhans J, Tempia S, Wolter N, von Gottberg A, Bhiman JN, Buys A, Moyes J, McMorrow ML, Kahn K, Xavier Gómez-Olivé F, Tollman S, Martinson NA, Wafawanaka F, Lebina L, du Toit J, Jassat W, Neti M, Brauer M, Cohen C, for the PHIRST-C Group. https://doi.org/10.1101/2021.05.26.21257849 doi: medRxiv preprint

11. Sabino EC, Buss LF, Carvalho MPS, Prete CA, Crispim MAE, Fraiji NA, et al. Resurgence of COVID-19 in Manaus, Brazil, despite high seroprevalence. Lancet (London, England). 2021;397:452-5. Available from: http://www.ncbi.n/m.nih.gov/pubmed/33515491 


\section{Figures/Tables}

Figure 1: Age and sex structure of surveyed donors, broken down by race and province (EC-Eastern Cape, FS-Free State, GP-Gauteng, LP-Limpopo, MP-Mpumalanga, NC-Northern Cape, NW-North West, WC-Western Cape, ZN-KwaZulu Natal)

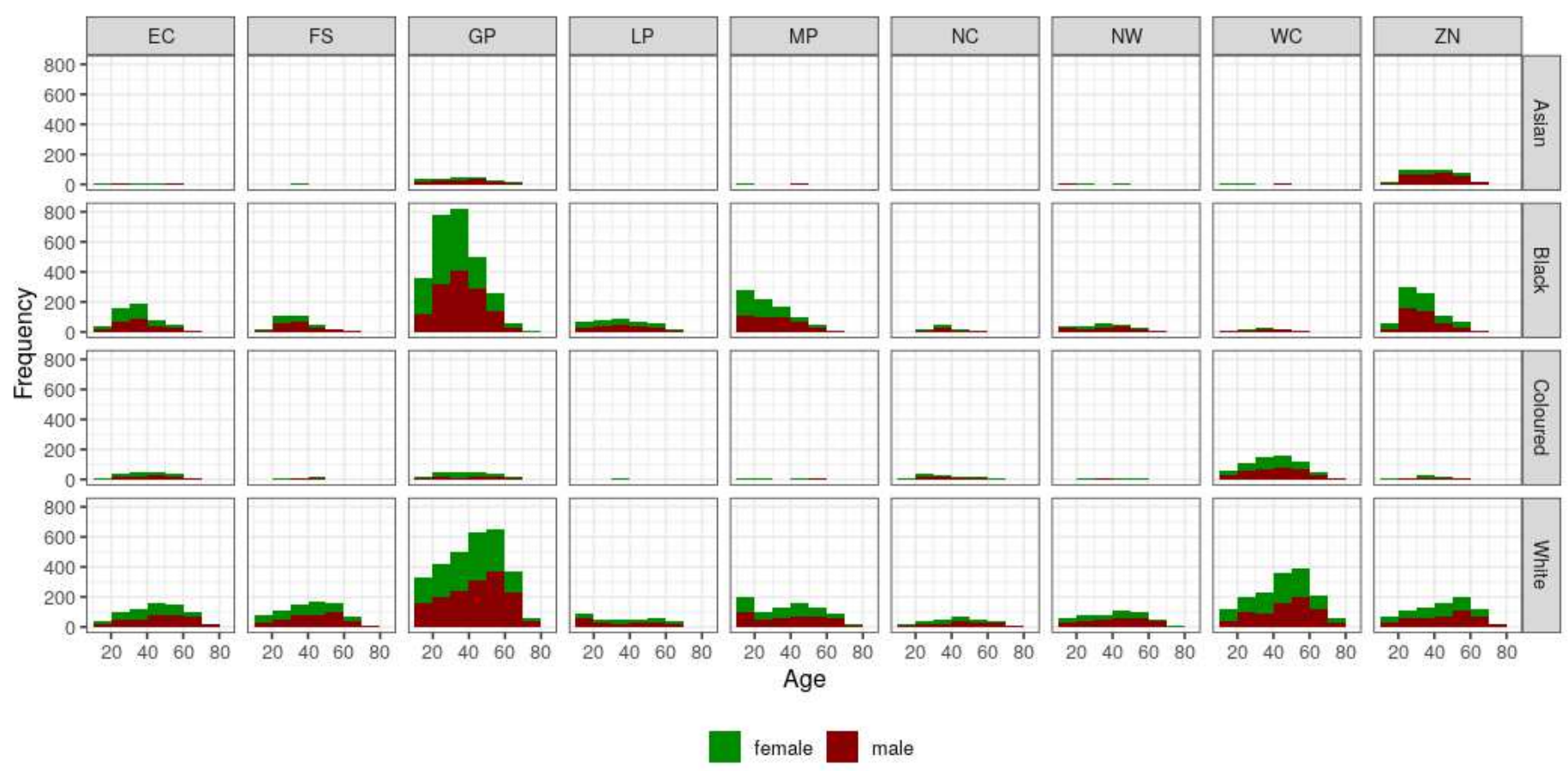


Table 1: The demographic breakdown of the blood donor population.

\begin{tabular}{|c|c|c|c|c|}
\hline & Reactive & Non-Reactive & Total & Crude Estimates (\%) \\
\hline \multicolumn{5}{|l|}{ Province } \\
\hline Eastern Cape & 569 & 896 & 1465 & 38.8 \\
\hline Free State & 289 & 793 & 1082 & 26.7 \\
\hline Gauteng & 1988 & 4216 & 6204 & 32.0 \\
\hline KwaZulu Natal & 663 & 1444 & 2107 & 31.5 \\
\hline Limpopo & 217 & 494 & 711 & 30.5 \\
\hline Mpumalanga & 563 & 1132 & 1695 & 33.2 \\
\hline Northern Cape & 100 & 367 & 467 & 21.4 \\
\hline North West & 202 & 530 & 732 & 27.6 \\
\hline Western Cape & 503 & 1796 & 2299 & 21.9 \\
\hline \multicolumn{5}{|l|}{ Race } \\
\hline Asian & 156 & 539 & 695 & 22.4 \\
\hline Black & 3155 & 2810 & 5965 & 52.9 \\
\hline Coloured & 448 & 908 & 1356 & 33.0 \\
\hline Unreported & 47 & 94 & 141 & 33.3 \\
\hline White & 1288 & 7317 & 8605 & 15.0 \\
\hline \multicolumn{5}{|l|}{ Sex } \\
\hline Female & 2567 & 5611 & 8178 & 31.4 \\
\hline Male & 2527 & 6057 & 8584 & 29.4 \\
\hline Totals & 5094 & 11668 & 16762 & 30.4 \\
\hline
\end{tabular}


Table 2: Total number of donors surveyed by province.

\begin{tabular}{|l|c|c|}
\hline \multicolumn{1}{|c|}{ Province } & Specimens & $\begin{array}{c}\text { Proportion } \\
\text { (\%) }\end{array}$ \\
\hline Eastern Cape & 1,465 & 8.7 \\
\hline Free State & 1,082 & 6.5 \\
\hline Gauteng & 6,202 & 37.0 \\
\hline Limpopo & 711 & 4.2 \\
\hline Mpumalanga & 1,695 & 10.1 \\
\hline Northern Cape & 467 & 2.8 \\
\hline Northwest & 732 & 4.4 \\
\hline Western Cape & 2,299 & 13.7 \\
\hline KwaZulu Natal & 2,107 & 12.6 \\
\hline Total & $\mathbf{1 6 , 7 6 2}$ & $\mathbf{1 0 0}$ \\
\hline
\end{tabular}


Figure 2a: Prevalence by age group, broken down by province and race for four provinces (ECEastern Cape, FS-Free State, GP-Gauteng, LP-Limpopo)

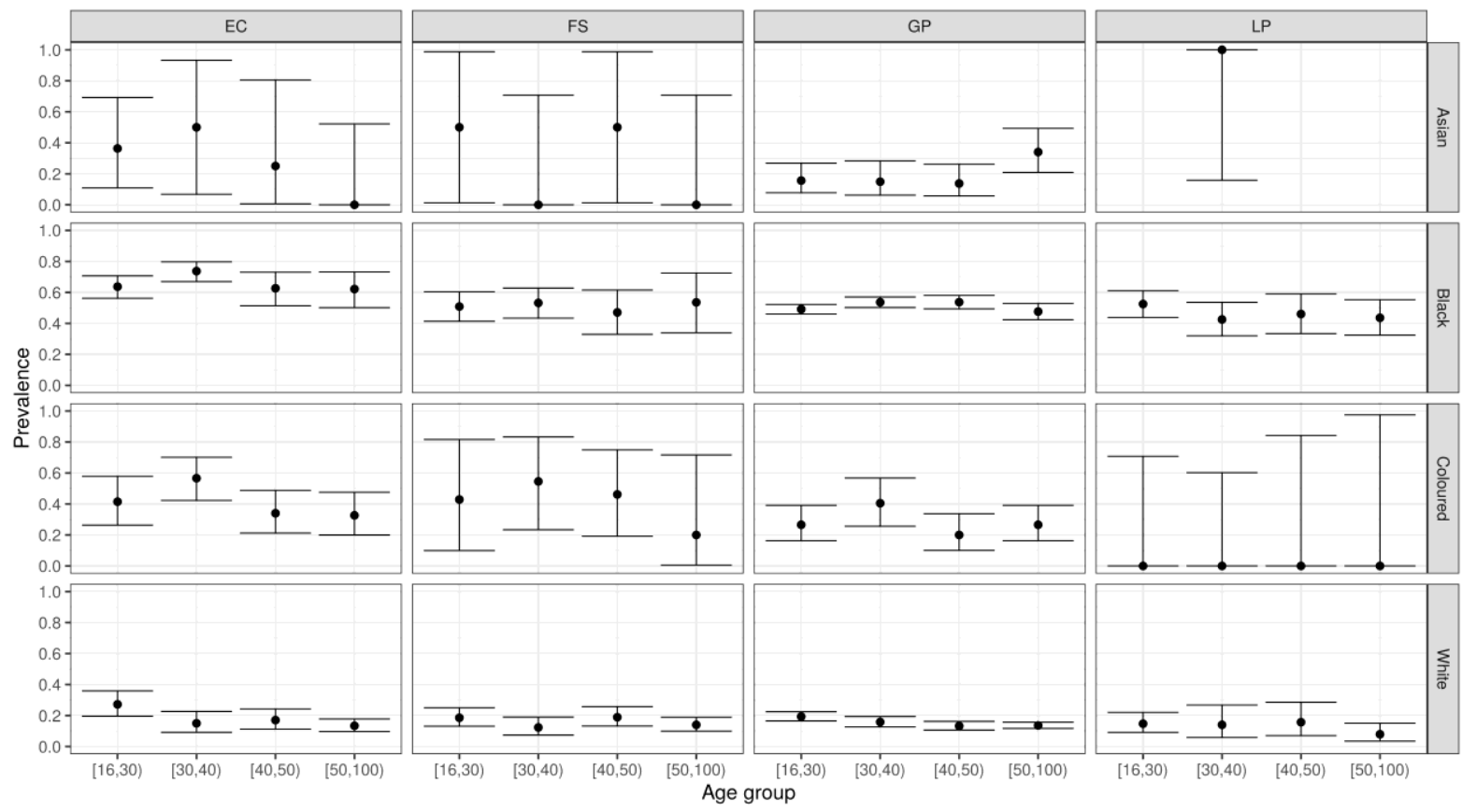

Figure 2b: Prevalence by age group, broken down by province and race for five provinces (MPMpumalanga, NC-Northern Cape, NW-North West, WC-Western Cape, ZN-KwaZulu-Natal)

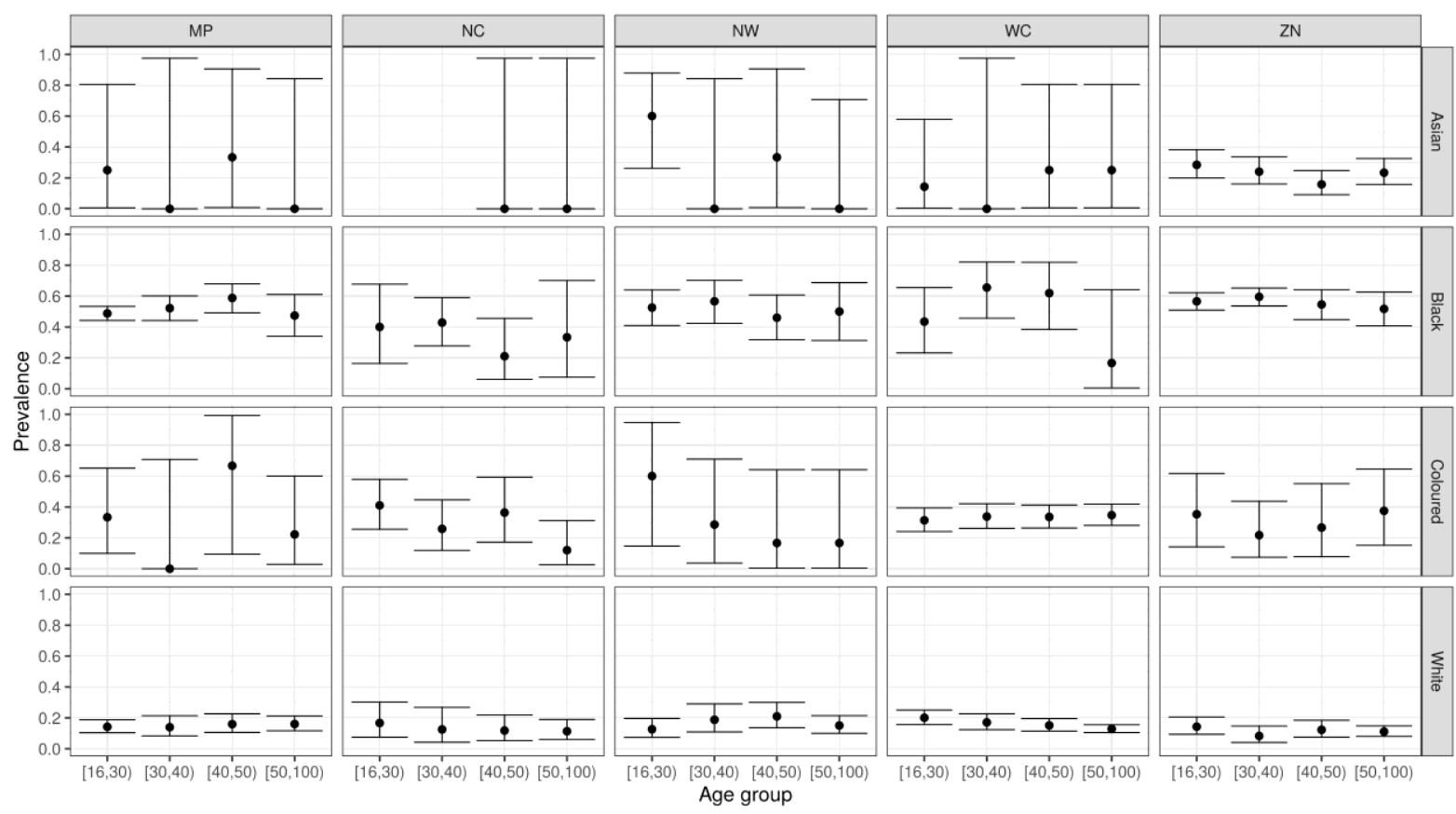


Figure 3: Prevalence comparison between sexes, by race and province (EC-Eastern Cape, FS-Free State, GP-Gauteng, LP-Limpopo, MP-Mpumalanga, NC-Northern Cape, NW-North West, WC-Western Cape, ZN-KwaZulu-Natal)

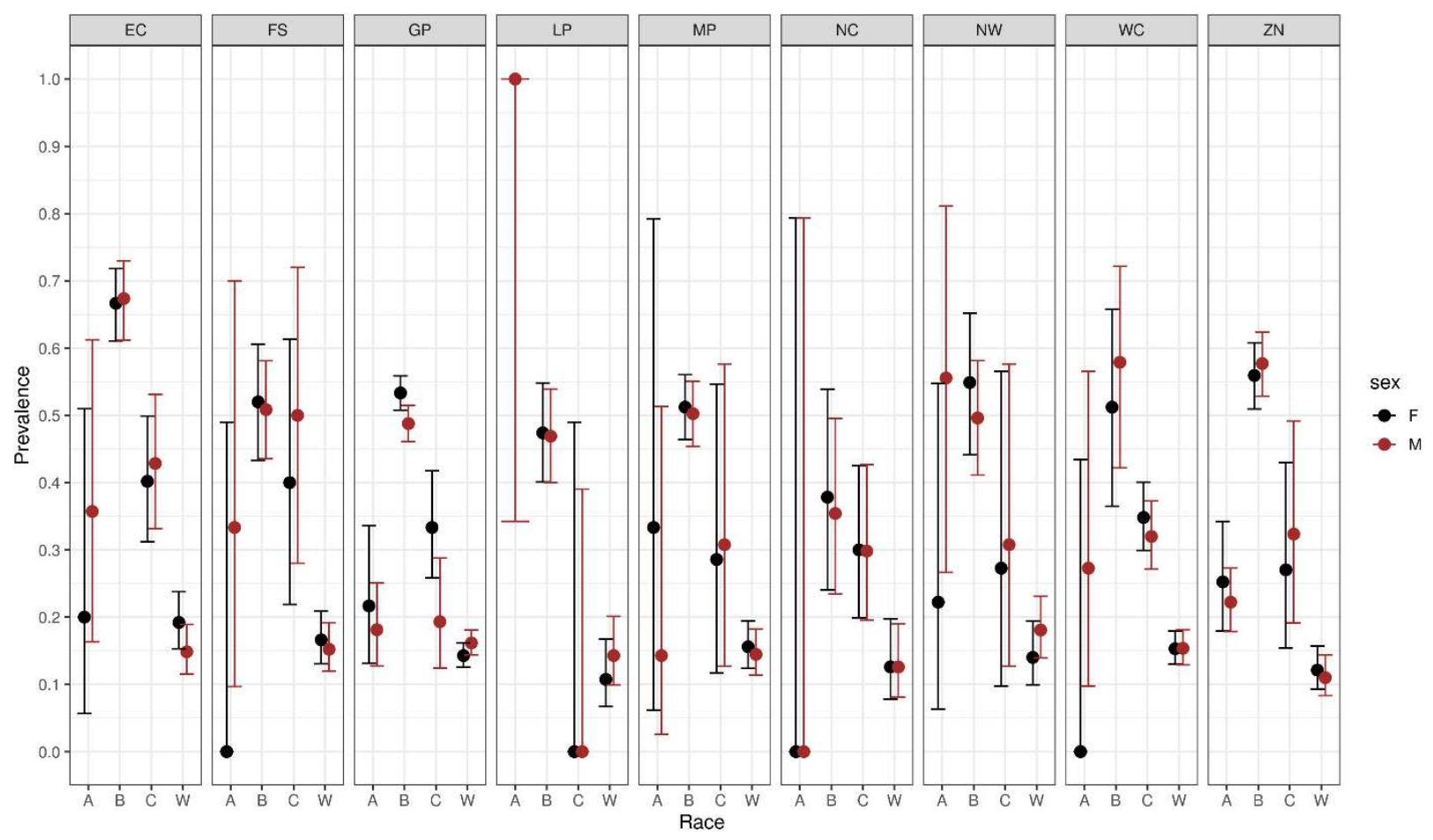


Figure 4: Prevalence by race (W-White, A-Asian, C-Coloured, B-Black) and province (EC-Eastern Cape, FS-Free State, GP-Gauteng, LP-Limpopo, MP-Mpumalanga, NC-Northern Cape, NW-North West, WCWestern Cape, ZN-KwaZulu-Natal), showing also the race weighted provincial estimates (Tot), and the prevalence implied by diagnosed cases reported to the National Institute for Communicable Diseases (Dx).

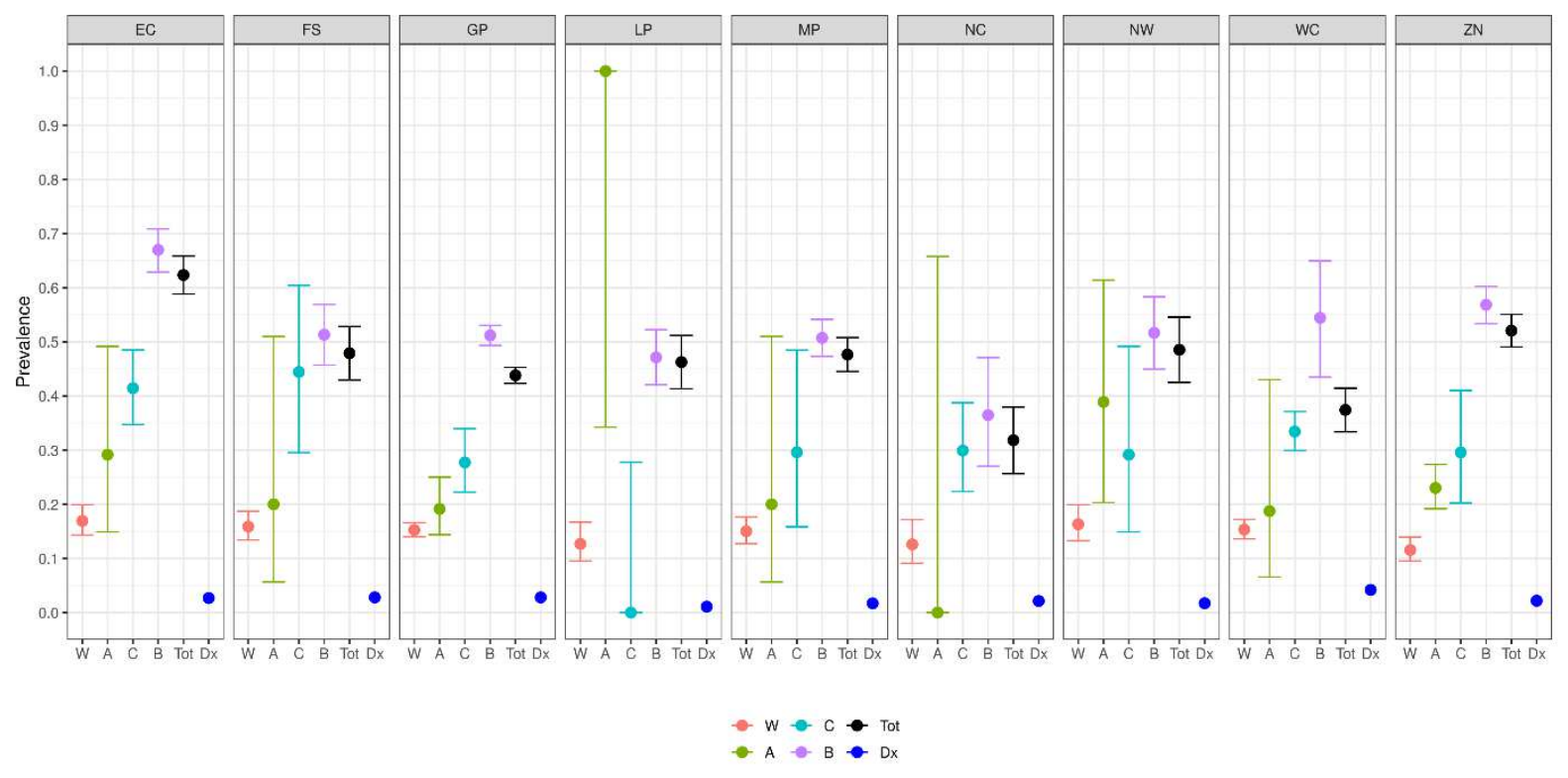


Table 3 Provincial estimates of prevalence; the implied number of infections; the number of laboratory confirmed cases; and the (multiplicative) discrepancy between our estimate and the official count.

\begin{tabular}{|l|r|r|r|r|}
\hline \multicolumn{1}{|c|}{ Province } & \multicolumn{1}{c|}{$\begin{array}{c}\text { Estimated } \\
\text { Prevalence } \\
(\%)\end{array}$} & \multicolumn{1}{c|}{$\begin{array}{c}\text { Estimated } \\
\text { Total } \\
\text { Infections }\end{array}$} & \multicolumn{1}{c|}{$\begin{array}{c}\text { Official } \\
\text { Diagnosed } \\
\text { Cases }\end{array}$} & $\begin{array}{c}\text { Diagnostic } \\
\text { Underestimate } \\
\text { (Fold) }\end{array}$ \\
\hline Eastern Cape & $62.5(58.8,65.9)$ & $2,724,350$ & 176,902 & 15.4 \\
\hline Free State & $47.8(42.8,53.0)$ & 925,093 & 81,622 & 11.3 \\
\hline Gauteng & $43.8(42.3,45.4)$ & $4,926,044$ & 434,495 & 11.3 \\
\hline Limpopo & $46.3(41.3,51.2)$ & $1,687,558$ & 64,966 & 26.0 \\
\hline Mpumalanga & $47.6(44.5,50.8)$ & $1,523,296$ & 81,758 & 18.6 \\
\hline Northern Cape & $31.8(25.7,38.0)$ & 235,156 & 25,007 & 9.4 \\
\hline Northwest & $48.5(42.5,54.6)$ & $1,302,318$ & 69,328 & 18.8 \\
\hline Western Cape & $37.4(33.4,41.4)$ & $1,855,484$ & 294,201 & 6.3 \\
\hline KwaZulu-Natal & $52.1(49.1,55.1)$ & $3,950,784$ & 249,703 & 15.8 \\
\hline
\end{tabular}

